\title{
Phenomenology of Higgs bosons in inverse seesaw model with Type-X two Higgs doublet at the LHC
}

\author{
Priyotosh Bandyopadhyay, ${ }^{a}$ Eung Jin $\mathrm{Chun}^{b}$ and Rusa Mandal ${ }^{c}$ \\ ${ }^{a}$ Indian Institute of Technology Hyderabad, Kandi, \\ Sangareddy-502287, Telengana, India \\ ${ }^{b}$ Korea Institute for Advanced Study, \\ Seoul 130-722, Korea \\ ${ }^{c}$ IFIC, Universitat de València-CSIC, \\ Apt. Correus 22085, E-46071 València, Spain \\ E-mail: bpriyo@iith.ac.in, ejchun@kias.re.kr, Rusa.Mandal@ific.uv.es
}

ABSTRACT: Type-X two Higgs doublet model is known to explain the muon $g-2$ anomaly with a relatively light charged Higgs boson at large $\tan \beta$. The light charged Higgs boson has been searched in the main $\tau \nu$ mode at the colliders. Invoking a scenario of inverse seesaw as the origin of neutrino masses and mixing, the charged Higgs boson can decay additionally to right-handed neutrinos which leads to interesting phenomenology. Considering generic lepton flavour violating signatures at the final states, a $5 \sigma$ discovery can be achieved with the early data of LHC, at $14 \mathrm{TeV}$, for relatively large inverse seesaw Yukawa coupling $Y_{N}$. The very light pseudoscalar and charged Higgs boson mass reconstruction are performed using the new modes and the results look promising. The inverse seesaw Yukawa coupling is shown to be probed down to $Y_{N} \sim 0.2$ at HL LHC with $3000 \mathrm{fb}^{-1}$.

KeYwords: Phenomenological Models

ARXIV EPRINT: 1904.09494 


\section{Contents}

1 Introduction 1

2 The model 2

3 Benchmark points $\quad \mathbf{5}$

3.1 Decay branching fractions 5

$\begin{array}{lll}3.2 & \text { Cross-section } & 7\end{array}$

$\begin{array}{lll}3.3 & \text { Final states } & 7\end{array}$

4 Collider simulation at the LHC 9

5 Results $\quad 11$

$5.12 \tau_{\text {jet }}+2 \ell \quad 11$

$\begin{array}{lll}5.2 & 2 \tau_{\text {jet }}+3 \ell & 14\end{array}$

$\begin{array}{lll}5.3 & \text { Very light pseudoscalar } & 14\end{array}$

6 Reconstruction of charged Higgs boson mass 15

$\begin{array}{lll}7 & \text { Conclusions } & 17\end{array}$

\section{Introduction}

Non-observation of flavour changing neutral currents classifies Two-Higgs-Doublet Models (2HDMs) to four different categories which differ by the pattern of Higgs doublets' interaction to fermions [1]. A discrete symmetry $Z_{2}$ is imposed on these models under which the Higgs doublets and fermions carry different parities. The well-known nomenclature is "Type-I", "Type-II", "lepton-specific" (or "Type-X") and "flipped" (or "Type-Y") 2HDMs. An interesting scenario is the Type-X $2 \mathrm{HDM}$ which can explain the $g-2$ anomaly [2] by evading all the collider bounds for high $\tan \beta$ regime [3-9]. An extension of such scenario with a scalar dark matter candidate also provides interesting signature in indirect measurements [10]. In this large $\tan \beta$ limit, due to the suppression in couplings of the heavy Higgs bosons to quarks (which affects their production cross section at the LHC), the popularity of this model is depreciated from collider searches point of view. An alleviation is possible in presence of a light pseudoscalar which opens the decay modes to $A, Z$ and $A, W^{ \pm}$for the heavier Higgs bosons, $H$ and $H^{ \pm}$, respectively. The decay width of $H^{ \pm} \rightarrow A W^{ \pm}$is independent of $\tan \beta$ and depends only on the gauge coupling, thus the branching fraction in this mode becomes very prominent at high $\tan \beta$ region where the other decay modes are suppressed. In the context of Type-X, the parameter space with a light pseudoscalar boson and larger values of $\tan \beta$ has been investigated in various direct and indirect searches [4-9]. 
This decay mode of charged Higgs has also been looked into for Type-I and Type-II 2HDM scenarios [11-13].

The presence of light pseudoscalar is very natural in $Z_{3}$ symmetric superpotential viz., NMSSM [14, 15] and Triplet-Singlet-extended MSSM [16-19], where it arises as pseudo-Nambu-Goldstone mode and the studies for the decay of charged Higgs to this light pseudoscalar are performed as well. The muti-lepton and multi-tau final states are also investigated in the context of triplet-like charged Higgs bosons with the bounds from $B \rightarrow X_{s} \gamma$ [20-22]. However, such studies do not have the right-handed neutrino (RHN) in the final states. Construction of the RHN thus becomes very crucial in order to distinguish our scenario from the rest. As an additional benefit, non-democratic lepton-flavour signature arise at the final states which is a smoking gun signature of our model.

The signature arising from the RHN can be enhanced at the colliders with a relatively larger Yukawa coupling of a RHN via inverse seesaw mechanism. This also enriches the phenomenology and the search for such Type-X charged Higgs boson. In an inverse seesaw framework the RHN is a pseudo-Dirac fermion allowing an $\mathcal{O}(1)$ coupling with the Higgs bosons. This enables us to search for the decays of the charged Higgs boson into charged lepton and RHN, where the RHN can further decay into the following modes: charged lepton/neutrino and gauge boson, neutrino and Higgs boson, as well as light pseudoscalar and neutrino. The right-handed neutrinos decays to charged Higgs can be seen in the context of other scenarios $[23,24]$ but can only be enhance in inverse-seesaw case due to relatively large Yukawa coupling In this article we are mostly interested in probing the decay modes with charged lepton, gauge boson and also the pseudoscalar, which is generic in Type X 2HDM, at the LHC.

The paper is organized as follows. In section 2 we briefly describe the model. By studying the parameter space allowed by several measurements, we chose the benchmark points in section 3. The collider phenomenology is discussed in section 4 and the corresponding results are presented in section 5 including a discussion with the phenomenology of light pseudoscalar which is different compared to the other benchmark cases. In section 6 we discuss the charged Higgs mass reconstruction and the reach at current and future LHC. Finally in section 7 we present the concluding remarks.

\section{The model}

We consider three generations of $N_{R}$ and $S_{2}$, the two Majorana neutrinos forming a pseudoDirac fermion, which are singlet under the SM gauge group. Here $N_{R}$ couples to the lefthanded active neutrino $\nu_{L}$ via Yukawa coupling $Y_{N}$ shown in eq. (2.1), which can be $\mathcal{O}(1)$ in the inverse seesaw mechanism [25-33]. The other Majorana neutrino $S_{2}$ does not have any direct coupling to the SM sectors but mixes with $N_{R}$ via a mass mixing term proportional to $M_{N}$ (the fifth term in eq. (2.1)). It has a Majorana mass term $\mu$ which can be very small motived from the breaking of higher $\mathrm{U}(1)_{B-L}$ gauge group [31, 32].

Here we invoke the inverse seesaw mechanism in the Type-X $2 \mathrm{HDM}$, which is capable in explaining the muon $g-2$ anomaly at $2 \sigma$ level in presence of a light pseudoscalar [2]. In this case the charged Higgs boson can also be very light unlike Type-II 2HDM, which 
suffers from indirect bounds arising from $B \rightarrow X_{s} \gamma$ [34] mode. In eq. (2.1) we see that the leptons interact to the Higgs doublet $\Phi_{1}$ whereas the quarks couple to $\Phi_{2}$. Interestingly, the RHN $N_{R}$ can couple to both $\Phi_{1}$ and $\Phi_{2}$ and we call such extensions as Type-X and Type- $\mathrm{X}^{\prime}$, respectively. In the succeeding sections we focus on Type-X parameter space for collider phenomenology.

$$
\begin{aligned}
-\mathcal{L}= & \left(Y_{u} \bar{Q}_{L} \tilde{\Phi}_{2} u_{R}+Y_{d} \bar{Q}_{L} \Phi_{2} d_{R}+Y_{l} \bar{\ell}_{L} \Phi_{1} e_{R}+Y_{N}^{(\prime)} \bar{\ell}_{L} \tilde{\Phi}_{1,2} N_{R}+M_{N} \bar{N}_{R}^{c} S_{2}+\text { h.c. }\right) \\
& +\mu \bar{S}_{2}^{c} S_{2}+V\left(\Phi_{1}, \Phi_{2}\right) .
\end{aligned}
$$

Note that $Y_{N}^{(\prime)}$ corresponds to $3 \times 3$ Yukawa matrix which couples the RHNs to different SM lepton generations. The Higgs doublets $\Phi_{1,2}$ are given by eq. (2.2) and $\tilde{\Phi}_{2}=i \sigma_{2} \phi_{2}^{*}$ where $\sigma_{2}$ is the Pauli matrix.

$$
\Phi_{1,2}=\left(\begin{array}{c}
\phi_{1,2}^{+} \\
\frac{1}{\sqrt{2}}\left(v_{1,2}+h_{1,2}+i a_{1,2}\right)
\end{array}\right) .
$$

The neutrino mass terms in the Lagrangian can be written as

$$
-\mathcal{L}_{m}^{\nu}=\mu \bar{S}_{2}^{c} S_{2}+m_{D} \bar{\nu}_{L} N_{R}+M_{N} \bar{N}_{R}^{c} S_{2}+\text { h.c. }
$$

where $m_{D}=Y_{N}^{(\prime)} v_{1,2} / \sqrt{2}$ for Type-X and Type- $\mathrm{X}^{\prime}$, respectively. In the basis of $\nu_{L}^{c}, N_{R}, S_{2}$, the $9 \times 9$ neutrino mass matrix takes the form as

$$
m_{\nu}=\left(\begin{array}{ccc}
0 & m_{D} & 0 \\
m_{D}^{T} & 0 & M_{N} \\
0 & M_{N}^{T} & \mu
\end{array}\right) .
$$

Diagonalizing the matrix (eq. (2.4)) we have three categories for neutrinos where the masses are given by

$$
\begin{aligned}
m_{\nu_{\ell}} & =m_{D} M_{N}^{-1} \mu\left(M_{N}^{T}\right) m_{D}^{T}, \\
m_{N_{H}}^{2} & =m_{N_{H^{\prime}}}^{2}=M_{N}^{2}+m_{D}^{2} .
\end{aligned}
$$

We designate these nearly mass degenerate Majorana eigenstates as $N_{i}$, where $i \in\{1-6\}$, for the rest of the paper.

Having two Higgs doublets $\Phi_{1,2}$, we write the $Z_{2}$ symmetric scalar potential as

$$
\begin{aligned}
V= & m_{11}^{2}\left|\Phi_{1}\right|^{2}+m_{22}^{2}\left|\Phi_{2}\right|^{2}-m_{12}^{2}\left(\Phi_{1}^{\dagger} \Phi_{2}+\Phi_{1} \Phi_{2}^{\dagger}\right) \\
& +\frac{\lambda_{1}}{2}\left|\Phi_{1}\right|^{4}+\frac{\lambda_{2}}{2}\left|\Phi_{2}\right|^{4}+\lambda_{3}\left|\Phi_{1}\right|^{2}\left|\Phi_{2}\right|^{2}+\lambda_{4}\left|\Phi_{1}^{\dagger} \Phi_{2}\right|^{2}+\frac{\lambda_{5}}{2}\left[\left(\Phi_{1}^{\dagger} \Phi_{2}\right)^{2}+\left(\Phi_{1} \Phi_{2}^{\dagger}\right)^{2}\right],
\end{aligned}
$$

where a (soft) $Z_{2}$ breaking term $m_{12}^{2}$ is introduced. Minimization of the scalar potential determines the vacuum expectation values $\left\langle\Phi_{1,2}^{0}\right\rangle=v_{1,2} / \sqrt{2}$ around which the Higgs doublet fields are expanded. The model contains five physical fields denoted by $H^{ \pm}, A, H$ and $h$ in the mass basis and their orthogonal combinations are the corresponding Goldstone 
modes $G^{ \pm, 0}$. The mass basis and gauge basis are related by the following rotation matrices

$$
\begin{aligned}
\left(\begin{array}{l}
H^{ \pm} \\
G^{ \pm}
\end{array}\right) & =\left(\begin{array}{cc}
\sin \beta & \cos \beta \\
-\cos \beta & \sin \beta
\end{array}\right)\left(\begin{array}{l}
H_{1}^{ \pm} \\
H_{2}^{ \pm}
\end{array}\right), \quad\left(\begin{array}{c}
A \\
G^{0}
\end{array}\right)=\left(\begin{array}{cc}
\sin \beta & \cos \beta \\
-\cos \beta & \sin \beta
\end{array}\right)\left(\begin{array}{l}
a_{1} \\
a_{2}
\end{array}\right), \\
\left(\begin{array}{l}
H \\
h
\end{array}\right) & =\left(\begin{array}{cc}
\cos \alpha & \sin \alpha \\
-\sin \alpha & \cos \alpha
\end{array}\right)\left(\begin{array}{l}
h_{1} \\
h_{2}
\end{array}\right),
\end{aligned}
$$

where the angle $\beta$ is defined as $t_{\beta} \equiv \tan \beta=v_{2} / v_{1}$. The neutral CP-even Higgs bosons are diagonalized such that $h(H)$ denotes the lighter (heavier) state.

The gauge interaction of the Higgs bosons $h$ and $H$ are given by $\mathcal{L}_{\text {gauge }} \simeq$ $g_{V} m_{V}\left(s_{\beta-\alpha} h+c_{\beta-\alpha} H\right) V V$ where $V=W^{ \pm}$or $Z$. In the case of $h$ being $125 \mathrm{GeV}$ Higgs boson, the SM limit corresponds to $s_{\beta-\alpha} \rightarrow 1$. Indeed, LHC finds $c_{\beta-\alpha} \ll 1$ in all the 2HDMs confirming the SM-like property of the $125 \mathrm{GeV}$ boson [35].

Normalizing the Yukawa couplings of the neutral bosons and a fermion $f$ by $m_{f} / v$ factor where $v=\sqrt{v_{1}^{2}+v_{2}^{2}}=246 \mathrm{GeV}$, we obtain the following couplings of the respective Yukawa terms.

$$
\begin{aligned}
\overline{q_{L}} H^{ \pm} q_{R}^{\prime} & :-i \frac{\sqrt{2} \cot \beta}{v}\left[-m_{u} \bar{d}_{L} H^{-} u_{R}+m_{d} \overline{u_{L}} H^{+} d_{R}+\text { h.c. }\right] \\
\overline{q_{L}} A q_{R} & :-\frac{\cot \beta}{v}\left[-m_{u} \overline{u_{L}} A u_{R}+m_{d} \bar{d}_{L} A d_{R}+\text { h.c. }\right], \\
\overline{q_{L}} h q_{R} & :-\frac{-i \cos \alpha}{v \sin \beta}\left[m_{u} \overline{u_{L}} h u_{R}+m_{d} \bar{d}_{L} h d_{R}+\text { h.c. }\right], \\
\overline{q_{L}} H q_{R} & :-\frac{-i \sin \alpha}{v \sin \beta}\left[m_{u} \overline{u_{L}} H u_{R}+m_{d} \bar{d}_{L} H d_{R}+\text { h.c. }\right], \\
\bar{\ell}_{L} H \ell_{R}: & \frac{-i m_{\ell} \cos \alpha}{v \cos \beta}\left[\bar{\ell}_{L} H \ell_{R}+\text { h.c. }\right] \\
\bar{\ell}_{L} h \ell_{R}: & \frac{i m_{\ell} \sin \alpha}{v \cos \beta}\left[\bar{\ell}_{L} H \ell_{R}+\text { h.c. }\right] \\
\bar{\ell}_{L} A \ell_{R}: & \frac{m_{\ell} \tan \beta}{v}\left[\bar{\ell}_{L} A \ell_{R}+\text { h.c. }\right], \\
\bar{\nu} H^{+} \ell_{R}: & \frac{-i m_{\ell} \tan \beta}{v}\left[\bar{\nu} H^{+} \ell_{R}+\text { h.c. }\right] .
\end{aligned}
$$

However, as we are interested in Type-X 2HDM, the choice of interaction term of the RHN with the Higgs doublets is very crucial. For that reason we consider two cases as mentioned before and is explicitly shown in eq. (2.10), where we name it Type-X extension when the RHN couples to $\tilde{\Phi}_{1}$, like the SM leptons, and Type- $\mathrm{X}^{\prime}$ when it couples to $\tilde{\Phi}_{2}$.

$$
-\mathcal{L}_{\text {int }}^{\text {Type-X }}=Y_{N} \bar{\ell}_{L} \tilde{\Phi}_{1} N_{R} \quad \text { and } \quad-\mathcal{L}_{\text {int }}^{\text {Type-X' }}=Y_{N}^{\prime} \bar{\ell}_{L} \tilde{\Phi}_{2} N_{R}
$$

Depending on the Type-X or Type- $\mathrm{X}^{\prime}$ extension, the decays of RHN will have very different behavior with $\tan \beta$ variation. Below we list the relevant couplings of RHN with the other fields present in the model where the set in eq. (2.11) is for the Type-X case and eq. (2.12) 
refers to Type- $\mathrm{X}^{\prime}$ extension.

$$
\begin{aligned}
& \bar{\ell}_{L} H^{-} N_{R}: i Y_{N} \sin \beta\left[\bar{\ell}_{L} H^{-} N_{R}+\text { h.c. }\right] \text {, } \\
& \bar{\nu}_{L} h N_{R}: \frac{i Y_{N} \sin \alpha}{\sqrt{2}}\left[\bar{\nu}_{L} h N_{R}+\text { h.c. }\right] \text {, } \\
& \bar{\nu}_{L} H N_{R}: \frac{-i Y_{N} \cos \alpha}{\sqrt{2}}\left[\bar{\nu}_{L} H N_{R}+\text { h.c. }\right] \text {, } \\
& \bar{\nu}_{L} A N_{R}: \frac{-Y_{N} \sin \beta}{\sqrt{2}}\left[\bar{\nu}_{L} A N_{R}+\text { h.c. }\right] \text {. } \\
& \bar{\ell}_{L} H^{-} N_{R}: i Y_{N}^{\prime} \cos \beta\left[\bar{\ell}_{L} H^{-} N_{R}+\text { h.c. }\right] \text {, } \\
& \bar{\nu}_{L} h N_{R}: \frac{-i Y_{N}^{\prime} \cos \alpha}{\sqrt{2}}\left[\bar{\nu}_{L} h N_{R}+\text { h.c. }\right] \text {, } \\
& \bar{\nu}_{L} H N_{R}: \frac{-i Y_{N}^{\prime} \sin \alpha}{\sqrt{2}}\left[\bar{\nu}_{L} H N_{R}+\text { h.c. }\right] \text {, } \\
& \bar{\nu}_{L} A N_{R}: \frac{-Y_{N}^{\prime} \cos \beta}{\sqrt{2}}\left[\bar{\nu}_{L} A N_{R}+\text { h.c. }\right] \text {. }
\end{aligned}
$$

It can be seen that in high $\tan \beta$ region, the decay modes $H^{ \pm} \rightarrow \ell_{L} N_{R}$ and $N_{R} \rightarrow A \nu_{L}$, which are of our special interests, are enhanced only in Type-X extension and thus we examine the Type-X extension with RHNs in the rest of the paper. We also note that the decay $H^{ \pm} \rightarrow A W^{ \pm}$is governed only by the weak gauge coupling $g_{2}$ in all $2 \mathrm{HDM}$ scenarios, however, due to the dependency of $H^{ \pm} \rightarrow \ell_{L} N_{R}$ on $\tan \beta$ values, the partial branching fraction for $H^{ \pm} \rightarrow A W^{ \pm}$may vary which has important consequences in collider studies explored in the subsequent sections.

\section{Benchmark points}

To probe the exotic decays of the other (apart from the SM like one) Higgs bosons, specially the charged Higgs boson we choose some benchmark points for collider study. The $\mu \rightarrow e \gamma$ bounds from MEG collaboration [36] can be avoided by choosing the diagonal Yukawa for the RHNs. The EWPT also is allowed in the alignment limit [37-39]. In principle for collider searches we can choose the Yukawa responsible for inverse seesaw, $Y_{N_{i}}$ of $\mathcal{O}(1)$. For the current study we choose $Y_{N_{i}}=0.5$ for the democratic benchmark points viz. BP1, $\mathrm{BP} 2$ and BP3. However, for BP4 we choose $Y_{N_{1}}=0.5, Y_{N_{2,3}}=0.1$. In table 1 we present the mass spectra and other relevant parameters for these different benchmark points for the collider study. Amidst of such points BP3 has a light pseudoscalar with $m_{A} \sim 50 \mathrm{GeV}$.

\subsection{Decay branching fractions}

As discussed in the introduction the light charged Higgs boson $<500 \mathrm{Gev}$ is still allowed for Type-X compared to Type-II 2HDM. For the given BPs, we have chosen a charged Higgs boson with mass of $250 \mathrm{GeV}$, which opens up a large parameter space explaing the muon $g-2$ deviation [3]. The pseudoscalar mass varies from $49.6 \mathrm{GeV}$ to $200 \mathrm{GeV}$ depending on the benchmark points. Table 2 present the decay branching fractions for the charged 


\begin{tabular}{|c|c|c|c|c|}
\hline $\begin{array}{c}\text { Benchmark } \\
\text { Points }\end{array}$ & BP1 & BP2 & BP3 & BP4 \\
\hline$m_{h}$ & 125.5 & 125.5 & 125.5 & 125.5 \\
\hline$m_{H}$ & 250.1 & 250.1 & 250.1 & 250.1 \\
\hline$m_{A}$ & 100.0 & 200.1 & 49.6 & 100.0 \\
\hline$m_{H^{ \pm}}$ & 250.1 & 250.1 & 250.1 & 250.1 \\
\hline$m_{N}$ & 98.0 & 100.0 & 100.0 & 100.0 \\
\hline$Y_{N_{1}}$ & 0.5 & 0.5 & 0.5 & 0.5 \\
\hline$Y_{N_{2,3}}$ & 0.5 & 0.5 & 0.5 & 0.1 \\
\hline $\tan \beta$ & 50.0 & 50.0 & 50.0 & 50.0 \\
\hline
\end{tabular}

Table 1. Benchmark points for a collider study consistent with $m_{h} \sim 125 \mathrm{GeV}$ the SM like Higgs mass and with the $2 \sigma$ limits of $h \rightarrow W W^{*}, Z Z^{*}, \gamma \gamma$ [35]. Here we have only considered the non-zero diagonal Yukawa couplings i.e., $Y_{N_{1,2,3}} \equiv Y_{N_{11,22,33}}$, respectively.

\begin{tabular}{|c|c|c|c|c|}
\hline $\begin{array}{c}\text { Benchmark } \\
\text { Points }\end{array}$ & BP1 & BP2 & BP3 & BP4 \\
\hline$A W^{ \pm}$ & 0.30 & 0.00 & 0.44 & 0.42 \\
\hline$\tau \nu_{\tau}$ & 0.22 & 0.34 & 0.17 & 0.33 \\
\hline$e^{ \pm} N$ & 0.16 & 0.22 & 0.13 & 0.23 \\
\hline$\mu^{ \pm} N$ & 0.16 & 0.22 & 0.13 & 0.01 \\
\hline$\tau^{ \pm} N$ & 0.16 & 0.22 & 0.13 & 0.01 \\
\hline
\end{tabular}

Table 2. Branching fraction for $H^{ \pm}$for collider study at the LHC for $Y_{N}=0.5\left(Y_{N_{1}}=0.5, Y_{N_{2,3}}=\right.$ 0.1) for BP1 - BP3 (BP4). Here $N$ corresponds to inclusive of heavy neutrinos, i.e., $\sum_{i} N_{i}$.

\begin{tabular}{|c|c|c|c|c|}
\hline $\begin{array}{c}\text { Benchmark } \\
\text { Points }\end{array}$ & BP1 & BP2 & BP3 & BP4 \\
\hline$\tau \bar{\tau}$ & 0.99 & 0.38 & 0.99 & 0.99 \\
\hline$\sum_{i} N_{i} \nu_{i}$ & 0.01 & 0.62 & 0.00 & $\sim 10^{-3}$ \\
\hline
\end{tabular}

Table 3. Branching fraction for $A$ for the benchmark points for collider study at the LHC.

Higgs bosons for the benchmark points. For all benchmark points except for BP2, we see that $A W^{ \pm}$is the dominant mode as for large $\tan \beta$ the $t b$ mode is suppressed which can be seen from eq. (2.9). Apart from $A W^{ \pm}$modes, the decay of charged Higgs boson to RHN and charged lepton can also be significant. For BP4, due of the choice of non-democratic Yukawa couplings i.e., $Y_{N_{1}}=0.5, Y_{N_{2,3}}=0.1$, the charged Higgs dominantly decays only to $N_{1} e^{ \pm}$states.

The light pseudoscalar mostly decays to tau anti-tau pair as shown in table. 3 . The $b \bar{b}$ mode is suppressed due to large value of $\tan \beta=50$ for all four benchmark points. However, as for BP2 $m_{A}=200 \mathrm{GeV}$, the branching fraction to $N \nu$ is $62 \%$ due to the available phase space compared to other BPs. For BP3, this mode is not kinematically allowed. 


\begin{tabular}{|c|c|c|c|c|}
\hline $\begin{array}{c}\text { Benchmark } \\
\text { Points }\end{array}$ & BP1 & BP2 & BP3 & BP4 \\
\hline$A Z$ & 0.26 & 0.00 & 0.41 & 0.38 \\
\hline$\tau \bar{\tau}$ & 0.24 & 0.33 & 0.19 & 0.36 \\
\hline$\sum_{i} N_{i} \nu_{i}$ & 0.50 & 0.67 & 0.39 & 0.26 \\
\hline
\end{tabular}

Table 4. Branching fraction for $H$ for the benchmark points for collider study at the LHC.

\begin{tabular}{|c|c|c|c|c|}
\hline $\begin{array}{c}\text { Benchmark } \\
\text { Points }\end{array}$ & BP1 & BP2 & BP3 & BP4 \\
\hline$W^{ \pm} \ell^{\mp}$ & 0.91 & 0.88 & & 0.91 \\
\hline$Z \nu$ & 0.09 & 0.12 & & 0.09 \\
\hline$A \nu$ & 0.00 & 0.00 & 1.00 & 0.00 \\
\hline
\end{tabular}

Table 5. Branching fraction for $N_{i}$ for the benchmark points for collider study at the LHC. Here $\ell^{\mp}$ spans over all three charged leptons namely, $e, \mu, \tau$ depending on the choice of $N_{i}$.

Finally we notice that the branching ratios for $H$ also changes compared to the 2HDM case as $N_{i} \nu_{i}$ modes are now open and have substantial branching fraction in this channel which can be read from table 4 . Due to the significant reduction in decay branching to $Z Z$ and $W^{ \pm} W^{\mp}$ final states, which are actually vanishing in this case, the heavy Higgs boson can easily evade the current bounds for various experimental searches [40, 41].

The RHNs in this case mostly decay to $W^{ \pm} \ell^{\mp}$ and the corresponding branching fraction is given in table 5 . The decays to final states with Higgs bosons are kinematically disallowed for all BPs and in the case of BP3, the RHNs decay completely to the light pseudoscalar and neutrino channel.

\subsection{Cross-section}

The model considered in this paper is implemented in SARAH [42] where the corresponding files for CalcHEP [43] are generated. The cross-sections for the Higgs bosons are calculated using CalcHEP with $\sqrt{\hat{s}}$ and CTEQ6L [44] are chosen as the renormalization and factorization scale and PDF, respectively. The largest cross-sections arise for $A H$ and $A H^{ \pm}$modes. The production cross-sections for $\mathrm{BP} 1$ and $\mathrm{BP} 4$ are the same as the mass spectrum and the Higgs couplings are the same. Below we discuss the final state topologies that can be probed at the LHC for the chosen benchmark points.

\subsection{Final states}

The final states which contain a RHN, $N_{i}$ are of our interest at the LHC. Due to singlet nature of RHN, it is difficult to produce them directly at the colliders viz., at the LHC. Thus such states can arise from either the decays of heavy neutral Higgs bosons $H$, the pseudoscalar $A$, or from the decays of the charged Higgs boson $H^{ \pm}$. The heavy neutral Higgs boson dominantly decays to $N_{i} \nu_{i}$ and the light pseudoscalar decays to $\tau \bar{\tau}$ and $N_{i} \nu_{i}$ depending on the available phase space. 


\begin{tabular}{|c|c|c|c|c|}
\hline $\begin{array}{c}\text { Benchmark } \\
\text { Points }\end{array}$ & BP1 & BP2 & BP3 & BP4 \\
\hline$A H$ & 26.8 & 11.5 & 39.5 & 26.8 \\
\hline$A H^{ \pm}$ & 49.7 & 21.8 & 72.8 & 49.7 \\
\hline$H H^{ \pm}$ & 14.7 & 14.7 & 14.7 & 14.7 \\
\hline$H^{ \pm} H^{\mp}$ & 8.1 & 8.1 & 8.1 & 8.1 \\
\hline
\end{tabular}

Table 6. Tree-level cross-section for the benchmark points obtained by CalcHEP [43] in the units of $\mathrm{fb}$ at the LHC with center of mass energy of $14 \mathrm{TeV}, \sqrt{\hat{s}}$ as renormalization and factorization scale, and CTEQ6L [44] as PDF.

The associated production of heavy Higgs boson along with pseudoscalar can have interesting decay topology as given in eq. (3.1). Given the mass spectrum for BP1 in table 1, the heavy Higgs can decay to $N_{i} \nu_{i}$ and the light pseudoscalar dominantly decays to tau anti-tau pair giving rise to di-tau plus opposite sign dilepton (OSD) final states as shown in eq. (3.1), where the leptons can be of different flavours. Thus it would be easy to distinguish the final state from the $Z$ boson contamination for the di-lepton.

$$
\begin{aligned}
p p & \rightarrow A H \rightarrow \tau \bar{\tau} N_{i} \nu_{i} \\
& \rightarrow \tau \bar{\tau} W^{ \pm} \ell_{i}^{\mp} \nu_{i} \\
& \rightarrow \tau \bar{\tau} \ell_{j}^{ \pm} \nu_{j} \ell_{i}^{\mp} \nu_{i},
\end{aligned}
$$

where $\ell_{i, j}^{ \pm}=e^{ \pm}, \mu^{ \pm}, \tau^{ \pm}$.

However, our main focus in this article is to probe the charged Higgs boson via its decay mode comprised of RHN, $N$. The light charged Higgs boson decays in the following kinematically allowed final states,

$$
\begin{aligned}
H^{ \pm} \rightarrow & \tau \nu \\
& e N \\
& A W^{ \pm} .
\end{aligned}
$$

If $m_{H^{ \pm}}>m_{N}$, then the produced charged Higgs can decay to $\ell^{ \pm} N$. Such RHN further decays via two-body or three-body decay to leptons and gauge bosons or leptons and jets, respectively. Thus for Type-X, where a very light charged Higgs boson is still allowed from the current LHC bounds [45-48] unlike the Type-II charged Higgs boson, we can explore such light charged Higgs boson by searching the final states given below in eqs. (3.3), (3.4), at the LHC. In this case, the dominant production mode is $p p \rightarrow A H^{ \pm}$, where the charged Higgs boson further decays into $N_{i} \ell_{i}^{ \pm}$given as

$$
\begin{aligned}
p p & \rightarrow A H^{ \pm} \rightarrow \tau \bar{\tau} N_{i} \ell_{i}^{ \pm} \\
& \rightarrow \tau \bar{\tau} W^{ \pm} \ell_{i}^{\mp} \ell_{i}^{ \pm} \\
& \rightarrow \tau \bar{\tau} \ell_{j}^{ \pm} \nu_{j} \ell_{i}^{\mp} \ell_{i}^{ \pm}
\end{aligned}
$$


where $\ell_{i, j}^{ \pm}=e^{ \pm}, \mu^{ \pm}, \tau^{ \pm}$. In collider only electron or muon can be detected as stable charged leptons giving rise to the following final state

$$
p p \rightarrow 2 \tau+2 e(2 \mu)+\mu(e)+\not p_{T} .
$$

The charged Higgs if decays to electron and RHN then it can give rise to signatures with different lepton flavours in final states as in the next step the RHN further decays to $e^{\mp} W^{ \pm}, Z \nu, h \nu$. As a result, we can have $2 \tau_{\text {jet }}+2 W^{ \pm}+2 e^{\mp}$ or $2 \tau_{\text {jet }}+W^{ \pm}+e^{\mp}+\left(\ell^{+} \ell^{-}\right)$. The interesting point to see that the gauge bosons decays to leptons via gauge coupling and so do not violate lepton flavours. Depending on the decays of RHN, we can have multi-leptonic final states with lepton flavour violation.

For the searches of single charged Higgs boson, the $b g$ fusion is still dominant $[15,49]$. In our case however, the final state lepton(s) can have different flavours $(e, \mu)$ owing to different branching ratios of Higgs boson to $e N$ and $\mu N$ due to non-democratic Yukawa for $\mathrm{BP} 4$.

\section{Collider simulation at the LHC}

For the chosen benchmark points we will focus on these non-standard decays of the charged Higgs boson as well as the other Higgs bosons. We use CalcHEP to calculate the cross-sections and the decay branching fractions from the benchmark points. The 'lhe' events are generated and fed to PYTHIA [50] for hadronization and fragmentation via the 'lhe' interface [51]. The simulation at hadronic level has been performed using the Fastjet-3.0.3 [52] with the CAMBRIDGE AACHEN algorithm. We have selected a jet size $R=0.5$ for the jet formation, with the following criteria:

- the calorimeter coverage is $|\eta|<4.5$

- the minimum transverse momentum of the jet $p_{T, \min }^{\text {jet }}=10 \mathrm{GeV}$ and jets are ordered in $p_{T}$

- leptons $(\ell=\mathrm{e}, \mu)$ are selected with $p_{T} \geq 10 \mathrm{GeV}$ and $|\eta| \leq 2.5$

- no jet should be accompanied by a hard lepton in the event

- $\Delta R_{\ell j} \geq 0.4$ and $\Delta R_{\ell \ell} \geq 0.2$

- Since an efficient identification of the leptons is crucial for our study, we additionally require a hadronic activity within a cone of $\Delta R=0.3$ between two isolated leptons to be $\leq 0.15 p_{T}^{\ell} \mathrm{GeV}$, with $p_{T}^{\ell}$ being the transverse momentum of the lepton, in the specified cone.

Equipped with the above set up and cuts we plot the lepton multiplicity $n_{\ell}$ and $p_{T}$ distribution in figure 1. Here the production process for the benchmark points is $p p \rightarrow$ $H^{+} H^{-}$. Such $H^{ \pm}$can decay to $\ell^{ \pm} N$ and the final state can have maximum of six charged leptons with non-universal lepton flavour number depending on the non-democratic Yukawa 

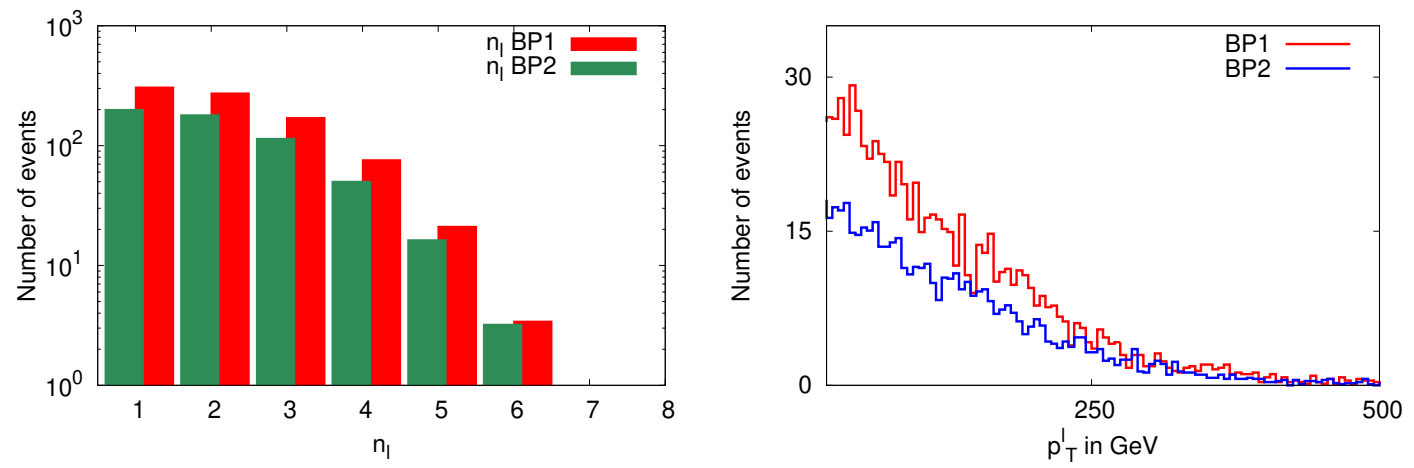

Figure 1. $n_{\ell}$ distribution (left panel) and $p_{T}^{\ell}$ distribution (right panel) for BP1 and BP2 at an integrated luminosity of $100 \mathrm{fb}^{-1}$ at the LHC with center of mass energy of $14 \mathrm{TeV}$.
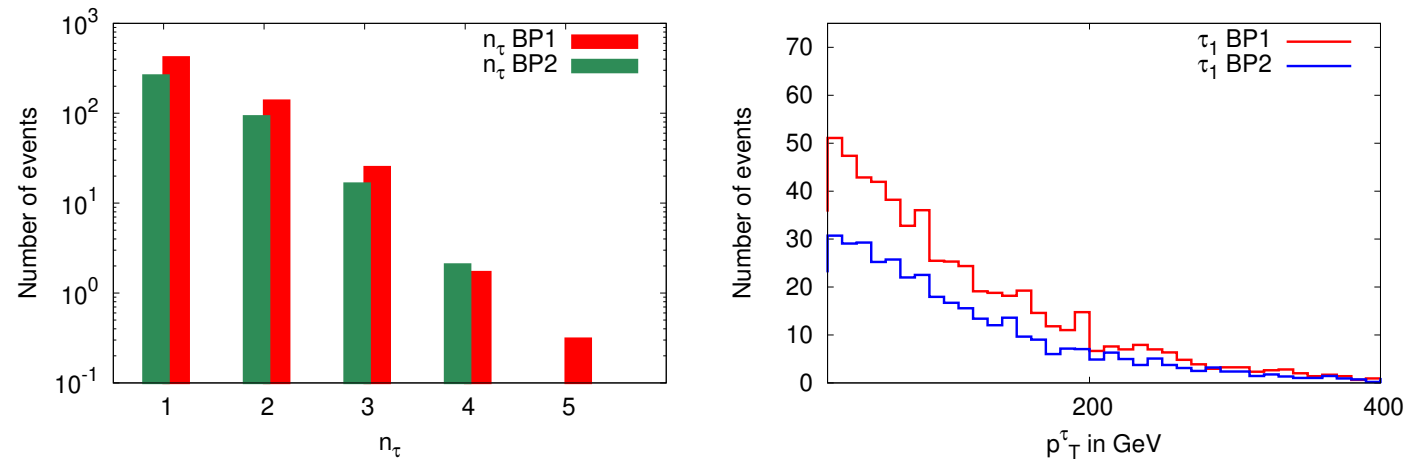

Figure 2. $n_{\tau_{\mathrm{jet}}}$ distribution (left panel) and $p_{T}^{\tau}$ distribution (right panel) for BP1 and BP2 at an integrated luminosity of $100 \mathrm{fb}^{-1}$ at the LHC with center of mass energy of $14 \mathrm{TeV}$.

coupling $Y_{N_{i}}$. Figure 1 (left panel) depicts that we can tag those multi-leptons as isolated charged leptons. In figure 1 (right panel) we show the $p_{T}^{\ell}$ distribution and some of them can actually be hard, as they may originate from the decay of the charged Higgs boson. Then there are relatively soft leptons arising from the $W^{ \pm}$decays. Finally the most soft charged leptons will come from the decay of the RHN $N_{i}$ due to smaller phase space for the decays to $\ell^{ \pm} W^{\mp}, Z \nu$ states.

Figure 2 describes the tau multiplicity $n_{\tau_{\text {jet }}}$ and $p_{T}^{\tau}$ distribution in left and right panels, respectively. The main source of the taus are from the decay of the pseudoscalar boson. The charged Higgs boson has sufficiently large branching fraction to $A W^{ \pm}$for $\mathrm{BP} 1, \mathrm{BP} 3$ and BP4, which can give rise to multi-tau signature along with the taus coming from the decays of the gauge bosons. For the $p_{T}^{\tau}$ distribution in figure 2, we only plot the events arising from the charged Higgs pair production. In the analysis we have considered all the production modes. The taus here are detected as hadronic tau jets $\tau_{\text {jet }}[53-55]$. The taus coming from the pseudoscalar can be hard depending on the mass of the pseudoscalar which can be noticed from the right panel. 


\section{Results}

In this section we present the event numbers for the final states for the benchmark points along with the dominant SM backgrounds. We focus on multi-tau and multi-lepton final states in which we also tag the lepton flavours in order to probe the inverse seesaw Yukawa coupling $Y_{N_{i}}$. In the first few subsections we discuss the results for BP1, BP2 and BP4, and the phenomenology for BP3 is discussed separately in subsection 5.3 due to the presence of light pseudoscalar boson.

\section{$5.12 \tau_{\text {jet }}+2 \ell$}

Table 7 presents the number of events for $2 \tau_{\text {jet }}+2 \ell, 2 \tau_{\text {jet }}+2 e, 2 \tau_{\text {jet }}+2 \mu$ and $2 \tau_{\text {jet }}+1 e+1 \mu$ respectively at the LHC with an integrated luminosity of $100 \mathrm{fb}^{-1}$. For the SM backgrounds we have considered all possible potential backgrounds in the analysis and only the non-zero ones are listed in the table. To be explicit, we calculated the following cases; $t \bar{t}, t \bar{t} Z, t \bar{t} W^{ \pm}$, $t Z W^{ \pm}, V V$ and $V V V$, where $V \in\left\{Z, W^{ \pm}\right\}$with all combinations.

The finalstate is reached in $\tau$ and we tag such $\tau$ s hadronically as $\tau_{\text {jet }}$ [53-55]. Here, in the case of the $\tau_{\text {jet }}$ we have considered the hadronic decay of the $\tau$ to be characterized by at least one charged track with $\Delta R \leq 0.1$ of the candidate $\tau_{\text {jet }}$ [53-55]. The demand of such hadronically reconstructed $\tau_{\text {jet }}$ along with the criteria of two isolated leptons reduce the SM background drastically. Given the finalstates with multi-leptons, $t \bar{t}$ and $t \bar{t} W^{ \pm}$seem to fail to contribute as backgrounds and the major contributions are expected to come from the di- and triple-gauge boson production including the $Z$ boson. However, mis-tagging of normal jets as tau-jets can contribute as SM backgrounds; especially for $t \bar{t}$ due it's large cross-section. For the completeness of the analysis we have considered a mis-tagging efficiency of $2 \%$, which is a conservative estimate for large $p_{T}$ tau-jets [56]. The finalstates $2 \tau+2 \ell$ (in table 7 ) and $2 \tau+3 \ell$ (in table 8 ) are affected by the mis-tagging efficiency. However, in table 9 such changes are insignificant.

The signal and the background numbers are subject to the uncertainties arising from the systematics as well as the statistics. Here we mainly focus on the systematics uncertainties and predict the range for signal significance in the succeeding paragraphs. The uncertainty in the cross-section is dominated by the PDF uncertainty which is around $10 \%$, then the jet-scale uncertainty is considered as $3 \%$ [55] and the tau-jet mis-tagging uncertainty is taken to be $8.8 \%$ [56]. In table 7 and table 8 the event numbers are given with their uncertainties for both the signal and backgrounds.

As mentioned earlier, for the considered benchmark points, dominant contribution arises from $A H^{ \pm}$production but other production processes are also significant. We see that for $2 \tau_{\text {jet }}+2 \ell$ channel, the minimum reach of $\mathrm{BP} 1, \mathrm{BP} 2$ and $\mathrm{BP} 4$ are $20.4 \sigma, 7.9 \sigma$ and $18.6 \sigma$, respectively. The signal significance denoted by $N_{\text {sig }}$ is calculated in a conservative approach as signal $/ \sqrt{\text { signal + background }}$.

The demand of only electron flavour can probe the non-democratic inverse seesaw Yukawa coupling $Y_{N_{i}}$ scenario. The final state of $2 \tau_{\text {jet }}+2 e$ reduces both the signal as well as the background numbers. The signal significance for the benchmark points reduces to $10.9 \sigma, 4.7 \sigma$ and $14.1 \sigma$ respectively for $\mathrm{BP} 1, \mathrm{BP} 2$ and $\mathrm{BP} 4$. 


\begin{tabular}{|c|c|c|c|c|c|c|c|c|c|}
\hline \multirow{2}{*}{\multicolumn{2}{|c|}{ Final states }} & \multicolumn{4}{|c|}{ Benchmark Points } & \multicolumn{4}{|c|}{ Backgrounds } \\
\hline & & \multirow{2}{*}{$\begin{array}{c}\text { BP1 } \\
240.7\end{array}$} & \multirow{2}{*}{$\begin{array}{l}\text { BP2 } \\
64.9\end{array}$} & \multirow{2}{*}{$\begin{array}{c}\text { BP3 } \\
207.6\end{array}$} & \multirow{2}{*}{$\begin{array}{c}\mathrm{BP} 4 \\
258.8\end{array}$} & $t \bar{t}$ & $t \bar{t} V$ & $t Z W^{ \pm}$ & $\begin{array}{c}V V / \\
V V V \\
\end{array}$ \\
\hline 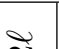 & $H A$ & & & & & \multirow{4}{*}{164.8} & \multirow{4}{*}{11.3} & \multirow{4}{*}{3.5} & \multirow{4}{*}{632.7} \\
\hline $\begin{array}{l}\sim \\
+\end{array}$ & $H^{ \pm} H^{\mp}$ & 22.8 & 23.6 & 13.7 & 53.0 & & & & \\
\hline$\stackrel{\vec{D}}{\circ}$ & $H H^{ \pm}$ & 182.2 & 53.6 & 267.5 & 131.6 & & & & \\
\hline & $A H^{ \pm}$ & 569.7 & 180.0 & 168.7 & 460.5 & & & & \\
\hline \multirow{2}{*}{\multicolumn{2}{|c|}{ Total }} & 1015.4 & 322.2 & 657.5 & 903.9 & \multirow{2}{*}{\multicolumn{4}{|c|}{$812.2 \pm 121.8$}} \\
\hline & & & & \pm 98.7 & \pm 135.6 & & & & \\
\hline \multicolumn{2}{|c|}{$N_{\text {sig }}($ in $\sigma)$} & $\{20.4,27.1\}$ & $\{7.9,11.4\}$ & $\{14.5,19.9\}$ & $\{18.6,25.0\}$ & & & & \\
\hline & $H A$ & 69.1 & 16.9 & 54.6 & 76.8 & \multirow{4}{*}{40.0} & \multirow{4}{*}{2.7} & \multirow{4}{*}{0.9} & \multirow{4}{*}{274.0} \\
\hline $\begin{array}{l}n \\
+ \\
+\end{array}$ & $H^{ \pm} H^{\mp}$ & 8.6 & 9.5 & 3.0 & 31.1 & & & & \\
\hline$\stackrel{\Delta}{.}$ & $H H^{ \pm}$ & 63.1 & 21.1 & 78.9 & 74.0 & & & & \\
\hline ล & $A H^{ \pm}$ & 185.7 & 71.5 & 52.8 & 272.4 & & & & \\
\hline \multicolumn{2}{|r|}{ Total } & $\begin{array}{l}326.5 \\
\pm 49.0\end{array}$ & $\begin{array}{l}119.0 \\
\pm 17.9\end{array}$ & $\begin{array}{l}189.3 \\
\pm 28.4\end{array}$ & $\begin{array}{l}454.3 \\
\pm 68.2\end{array}$ & \multicolumn{4}{|c|}{$317.7 \pm 47.7$} \\
\hline \multicolumn{2}{|c|}{$N_{\text {sig }}($ in $\sigma)$} & $\{10.9,14.8\}$ & $\{4.7,6.8\}$ & $\{7.0,9.9\}$ & $\{14.1,18.6\}$ & & & & \\
\hline & $H A$ & 75.5 & 17.6 & 51.7 & 67.9 & \multirow{4}{*}{42.0} & \multirow{4}{*}{4.7} & \multirow{4}{*}{1.4} & \multirow{4}{*}{328.7} \\
\hline $\begin{array}{l}N \\
+\end{array}$ & $H^{ \pm} H^{\mp}$ & 9.0 & 9.7 & 3.7 & 9.3 & & & & \\
\hline$\stackrel{\overrightarrow{0}}{\circ}$ & $H H^{ \pm}$ & 69.0 & 22.7 & 78.6 & 26.8 & & & & \\
\hline$\stackrel{t}{N}$ & $A H^{ \pm}$ & 195.6 & 78.8 & 46.3 & 76.2 & & & & \\
\hline \multicolumn{2}{|r|}{ Total } & $\begin{array}{l}349.1 \\
\pm 52.4\end{array}$ & $\begin{array}{l}128.8 \\
\pm 19.3\end{array}$ & $\begin{array}{c}180.3 \\
\pm 27.0\end{array}$ & $\begin{array}{l}180.2 \\
\pm 27.0\end{array}$ & & 376. & $8 \pm 56.5$ & \\
\hline & $i g($ in $\sigma)$ & $\{11.0,14.9\}$ & $\{4.7,6.8\}$ & $\{6.3,9.0\}$ & $\{6.3,9.0\}$ & & & & \\
\hline$\frac{2}{1}$ & $H A$ & 110.9 & 30.4 & 103.6 & 128.5 & & & & \\
\hline o & $H^{ \pm} H^{\mp}$ & 8.1 & 8.3 & 7.5 & 20.7 & & & & \\
\hline+ & $H H^{ \pm}$ & 81.0 & 16.8 & 148.8 & 51.2 & 82.4 & 3.8 & 1.3 & 30.4 \\
\hline$\stackrel{\leftrightarrow}{\mathrm{N}}$ & $A H^{ \pm}$ & 251.3 & 56.6 & 85.8 & 167.6 & & & & \\
\hline & & 451.4 & 112.1 & 345.7 & 368.0 & & & & \\
\hline & Total & \pm 67.7 & \pm 16.8 & \pm 51.9 & \pm 55.2 & & & 11 & \\
\hline$N_{s i}$ & $i g($ in $\sigma)$ & $\{16.8,20.9\}$ & $\{6.3,8.5\}$ & $\{14.2,17.8\}$ & $\{14.8,18.5\}$ & & & & \\
\hline
\end{tabular}

Table 7. The number of events for $2 \tau_{\text {jet }}+2 \ell$ final state at $100 \mathrm{fb}^{-1}$ of integrated luminosity at the LHC with $14 \mathrm{TeV}$ center of mass energy. The range for $N_{\text {sig }}$ is calculated incorporating the systematic uncertainties in signal and background events as well. The flavour tagging $(e, \mu)$ has been implemented. 

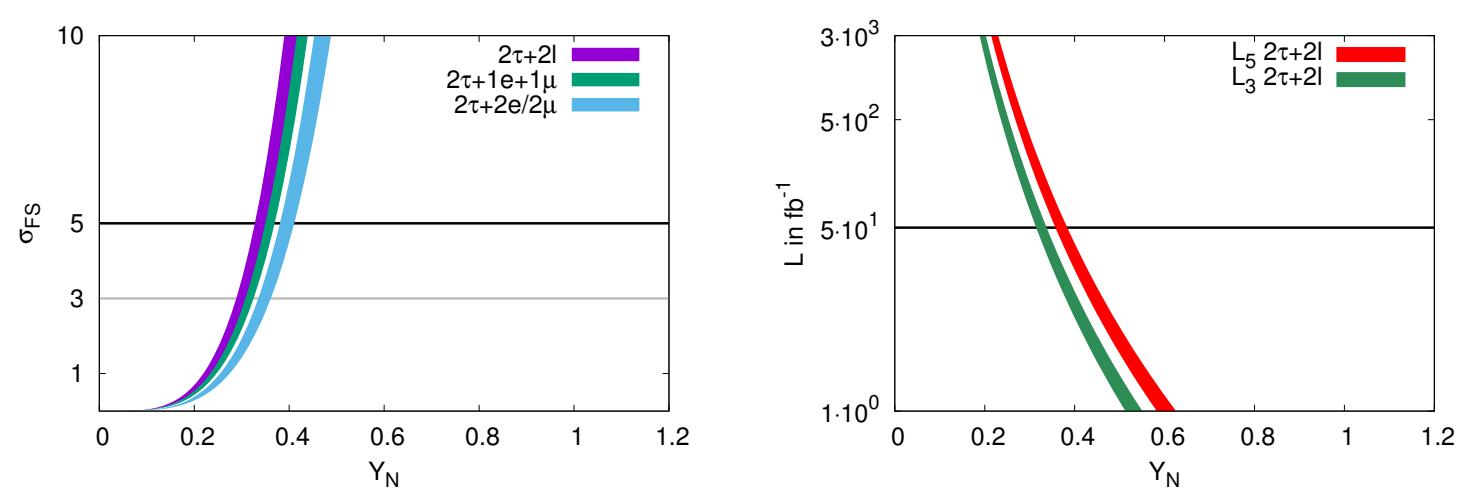

Figure 3. (Left panel) We present the signal significance verses $Y_{N}$ for the chosen final states at an integrated luminosity of $100 \mathrm{fb}^{-1}$. (Right panel) The required luminosity for $5 \sigma$ signal significance $\left(L_{5}\right)$ verses $Y_{N}$ are shown for $2 \tau+2 \ell$ final state with center of mass energy of $14 \mathrm{TeV}$ at the LHC.

Next we look at the final state having $2 \tau_{\text {jet }}+2 \mu$ where for BP1 and BP2 have event number similar to $2 \tau_{\text {jet }}+2 e$ channel as they have democratic inverse seesaw Yukawa coupling $Y_{N_{i}}$. However, in BP4, the number of event for $2 \tau_{\text {jet }}+2 \mu$ reduces substantially due to nondemocratic choice $Y_{N_{1}}=0.5, Y_{N_{2,3}}=0.1$. The charged Higgs boson as well CP-even heavy Higgs boson decay to $A W^{ \pm}$and $A Z$ for $\mathrm{BP} 4$, which contributes to di-muon final state. The respective minimum signal significance for $\mathrm{BP} 1, \mathrm{BP} 2$ and $\mathrm{BP} 4$ are $11.0 \sigma, 4.7 \sigma$ and $6.3 \sigma$, which is lower only for $\mathrm{BP} 4$ with respect to the $2 \tau_{\text {jet }}+2 e$ final state.

Finally we also present the event numbers for $2 \tau_{\text {jet }}+1 e+1 \mu$ final states and the corresponding minimum signal significances are $16.8 \sigma, 6.3 \sigma$ and $14.8 \sigma$ for $\mathrm{BP} 1, \mathrm{BP} 2$ and $\mathrm{BP} 4$, respectively.

It can be seen from the above discussion that $2 \tau+2 \ell$ final states have very high signal significance for all BPs. We use these modes to explore the reach to probe Yukawa coupling $Y_{N}$ at the LHC with center of mass energy of $14 \mathrm{TeV}$. The result is depicted in figure 3. The left panel shows the variation of signal significances w.r.t. the Yukawa coupling $Y_{N}$, where purple, green and blue bands correspond to the $2 \tau+2 \ell, 2 \tau+1 e+1 \mu$ and $2 \tau+2 e / 2 \mu$ final states respectively for an integrated luminosity of $100 \mathrm{fb}^{-1}$. The horizontal gray line corresponds to the signal significance of $3 \sigma$ over SM backgrounds, whereas the black line corresponds to $5 \sigma$ significance. It is evident that the inclusive $2 \tau+2 \ell$ has the maximum signal significance and the RHN Yukawa coupling $Y_{N} \gtrsim 0.3$ (within $15 \%$ systematic uncertainty as shown in the bands) for inverse seesaw can be probed with early data.

The table 7 result is then used to obtain the contour plots in figure 3 right panel for the signal significance in the plane spanned by integrated luminosity and the inverse seesaw Yukawa coupling $Y_{N}$. Here we present the contours of $3 \sigma$ and $5 \sigma$ significance by green and red bands, respectively, for the signal $2 \tau+2 \tau$ at the LHC with $14 \mathrm{TeV}$ center of mass energy, in the integrated luminosity verse Yukawa coupling $Y_{N}$ plane. We can see that, within $15 \%$ systematic uncertainty, $Y_{N} \sim 0.5$ and $\sim 0.6$ can receive $3 \sigma$ and $5 \sigma$ discovery respectively. For lower values of $Y_{N}$ we need higher integrated luminosity. For $\mathcal{O}(100) \mathrm{GeV}$ RHN mass, the LHC at $3000 \mathrm{fb}^{-1}$ can probe the inverse seesaw Yukawa coupling $\sim 0.2$. 


\section{$5.22 \tau_{\text {jet }}+3 \ell$}

Motivated by the topologies as described in eq. (3.1) and in eq. (3.3) we look for $3 \ell$ final state in association with $2 \tau_{\text {jet }}$. Obviously, the demand of $3 \ell$ reduces the SM backgrounds to negligible order. Table 8 present the number of events at the LHC with $14 \mathrm{TeV}$ center of mass energy at an integrated luminosity of $100 \mathrm{fb}^{-1}$.

The inclusive $2 \tau_{\text {jet }}+3 \ell$ final state has a minimum signal significance of $12 \sigma, 7.5 \sigma$ and $11.6 \sigma$ respectively for $\mathrm{BP} 1, \mathrm{BP} 2$ and $\mathrm{BP} 4.2 \tau_{\text {jet }}+2 e+1 \mu$ signal has significance of $6.9 \sigma, 3.8 \sigma$ and $7.6 \sigma$ respectively for $\mathrm{BP} 1, \mathrm{BP} 2$ and $\mathrm{BP} 4$. If we tag events with di-muon we find $2 \tau_{\text {jet }}+2 \mu+1 e$ with signal significance of $7.0 \sigma, 4.1 \sigma$ and $5.1 \sigma$ respectively for BP1, BP2 and $\mathrm{BP} 4$. As before for $\mathrm{BP} 4$, the significance drops down from the $2 e$ case due to nondemocratic inverse seesaw Yukawa $Y_{N_{i}}$. Such scenario can lead to experimental signature of lepton flavour violation in the final states $[24,33,57,58]$.

\subsection{Very light pseudoscalar}

As a consequent of very light pseudoscalar Higgs boson $\left(m_{A} \sim 50 \mathrm{GeV}\right)$, BP3 possess very different phenomenology compared to the other three benchmark points as the RHN completely decays to light pseudoscalar and light neutrinos (table 5). The $H^{ \pm}$and $H$ contribute to the RHN final states with $\sim 40 \%$ branching ratio. The final states searched in the previous subsections namely $2 \tau_{\text {jet }}+2 \ell$ and $2 \tau_{\text {jet }}+3 \ell$ also provide quite reasonable significance for BP3 as can be noted from table 7 and table 8, respectively, for all channels. Apart from these modes, we can also explore the final states comprised of RHN, with the topologies given in eqs. (5.1)-(5.4).

$$
\begin{aligned}
H^{ \pm} H & \rightarrow N e^{ \pm} N \nu \\
& \rightarrow 2 A+e^{ \pm}+3 \nu \\
& \rightarrow 4 \tau+e^{ \pm}+\not p_{T} \\
H^{ \pm} H^{\mp} & \rightarrow N e^{+} N e^{-} \\
& \rightarrow 4 \tau+O S E+\not p_{T} \\
A H & \rightarrow \tau \tau N \nu \\
& \rightarrow 4 \tau+\not p_{T} \\
A H^{ \pm} & \rightarrow \tau \tau N e^{ \pm} \\
& \rightarrow 4 \tau+e^{ \pm}+\not p_{T}
\end{aligned}
$$

The signal and non-zero background numbers are shown in table 9 for all channels. For BP3 the RHNs decay completely to the $A \nu$ states, and further decay of $A$ to tau pairs enrich the $4 \tau$ signature here. BP1, BP4 also compete with BP3 in these cases when produced in association with one pseudoscalar boson, which decays almost completely to tau pairs as well. We find $15.4 \sigma, 4.9 \sigma, 12.2 \sigma$ and $12.5 \sigma$ significance in $4 \tau_{\text {jet }}+\not p_{T} \geq 30 \mathrm{GeV}$ mode for $\mathrm{BP} 1, \mathrm{BP} 2, \mathrm{BP} 3$ and $\mathrm{BP} 4$, respectively. For $4 \tau_{\text {jet }}+1 e$ and $4 \tau_{\text {jet }}+1 \mu$ modes as no background events are observed, we use Poisson distribution to impose exclusion limits in the respective channels. It can be seen that for $\mathrm{BP} 1, \mathrm{BP} 3$ and $\mathrm{BP} 4$ the limits are just 


\begin{tabular}{|c|c|c|c|c|c|c|c|c|c|}
\hline \multirow{2}{*}{\multicolumn{2}{|c|}{ Final states }} & \multicolumn{4}{|c|}{ Benchmark Points } & \multicolumn{4}{|c|}{ Backgrounds } \\
\hline & & \multirow{2}{*}{$\begin{array}{l}\text { BP1 } \\
24.2\end{array}$} & \multirow{2}{*}{$\begin{array}{c}\text { BP2 } \\
0.0\end{array}$} & \multirow{2}{*}{$\begin{array}{r}\text { BP3 } \\
4.8\end{array}$} & \multirow{2}{*}{$\begin{array}{l}\text { BP4 } \\
23.9\end{array}$} & $t \bar{t}$ & $t \bar{t} V$ & $t Z W^{ \pm}$ & $\begin{array}{l}V V / \\
V V V\end{array}$ \\
\hline & $H A$ & & & & & \multirow{4}{*}{0.2} & \multirow{4}{*}{0.1} & \multirow{4}{*}{0.0} & \multirow{4}{*}{0.9} \\
\hline $\begin{array}{l}m \\
+\end{array}$ & $H^{ \pm} H^{\mp}$ & 5.6 & 6.4 & 0.9 & 13.5 & & & & \\
\hline 总 & $H H^{ \pm}$ & 43.0 & 13.5 & 50.2 & 32.8 & & & & \\
\hline$\curvearrowright$ & $A H^{ \pm}$ & 97.8 & 47.0 & 21.1 & 91.0 & & & & \\
\hline \multicolumn{2}{|c|}{ Total } & $\begin{array}{l}170.6 \\
\pm 25.6\end{array}$ & $\begin{array}{c}66.8 \\
\pm 10.0\end{array}$ & $\begin{array}{c}77.0 \\
\pm 11.6\end{array}$ & $\begin{array}{l}161.1 \\
\pm 24.2\end{array}$ & \multicolumn{4}{|c|}{$1.2 \pm 0.2$} \\
\hline \multicolumn{2}{|c|}{$N_{s i g}($ in $\sigma)$} & $\{12.0,14.0\}$ & $\{7.5,8.7\}$ & $\{8.0,9.4\}$ & $\{11.6,13.6\}$ & & & & \\
\hline$\frac{1}{3}$ & $H A$ & 6.6 & 0.00 & 1.7 & 6.9 & \multirow{4}{*}{0.1} & \multirow{4}{*}{0.0} & \multirow{4}{*}{0.0} & \multirow{4}{*}{0.5} \\
\hline$\stackrel{\sim}{N}$ & $H^{ \pm} H^{\mp}$ & 1.3 & 2.4 & 0.3 & 6.2 & & & & \\
\hline+ & $H H^{ \pm}$ & 13.9 & 2.8 & 19.0 & 13.1 & & & & \\
\hline$\stackrel{\mathrm{H}}{\stackrel{\mathrm{N}}{2}}$ & $A H^{ \pm}$ & 35.1 & 12.6 & 9.9 & 41.7 & & & & \\
\hline \multirow{2}{*}{\multicolumn{2}{|c|}{ Total }} & 53.3 & 17.8 & 30.9 & 67.9 & \multirow{2}{*}{\multicolumn{4}{|c|}{$0.6 \pm 0.1$}} \\
\hline & & \pm 8.5 & \pm 2.7 & \pm 4.6 & \pm 10.2 & & & & \\
\hline \multicolumn{2}{|c|}{$N_{s i g}($ in $\sigma)$} & $\{6.9,8.1\}$ & $\{3.8,4.4\}$ & $\{5.1,5.9\}$ & $\{7.6,8.8\}$ & & & & \\
\hline $\begin{array}{c}0 \\
+\end{array}$ & $H A$ & 8.1 & 0.0 & 0.8 & 7.4 & \multirow{4}{*}{0.1} & \multirow{4}{*}{0.0} & \multirow{4}{*}{0.0} & \multirow{4}{*}{0.2} \\
\hline$\frac{4}{3}$ & $H^{ \pm} H^{\mp}$ & 1.6 & 1.6 & 0.3 & 1.9 & & & & \\
\hline+ & $H H^{ \pm}$ & 17.1 & 4.1 & 19.8 & 7.2 & & & & \\
\hline$\stackrel{\leftrightarrow}{\stackrel{\leftrightarrow}{N}}$ & $A H^{ \pm}$ & 31.5 & 14.3 & 6.3 & 14.2 & & & & \\
\hline \multirow{2}{*}{\multicolumn{2}{|c|}{ Total }} & 58.3 & 20.0 & 27.2 & 30.8 & \multirow{2}{*}{\multicolumn{4}{|c|}{$0.3 \pm 0.0$}} \\
\hline & & \pm 8.7 & \pm 3.0 & \pm 4.1 & \pm 4.6 & & & & \\
\hline & $i g($ in $\sigma)$ & $\{7.0,8.2\}$ & $\{4.1,4.8\}$ & $\{4.8,5.6\}$ & $\{5.1,5.9\}$ & & & & \\
\hline
\end{tabular}

Table 8. The number of events for $2 \tau_{\text {jet }}+3 \ell$ final state at $100 \mathrm{fb}^{-1}$ of integrated luminosity at the LHC with $14 \mathrm{TeV}$ center of mass energy. The range for $N_{\text {sig }}$ is calculated incorporating the systematic uncertainties in signal and background events as well. The flavour tagging $(e, \mu)$ has been implemented.

below $2 \sigma$ level, however for BP2 these two contributions are suppressed as the pseudoscalar mostly decays to $N \nu$ states (with branching fraction $62 \%$ given in table 3 ). The channels with $4 \tau_{\text {jet }}+2 e$ and $4 \tau_{\text {jet }}+2 \mu$ are not at satisfactory level for $100 \mathrm{fb}^{-1}$ luminosity and we do not calculate the signal significance for these low signal event numbers and one needs to wait for more data for such prediction.

\section{Reconstruction of charged Higgs boson mass}

In this section we probe the $A H^{ \pm}$production mode which follows the following decay chain leading to $2 \tau+2 j+2 \ell$ final state.

$$
A H^{ \pm} \rightarrow \tau^{+} \tau^{-} N_{i} \ell^{ \pm} \rightarrow 2 \tau+\ell^{ \pm} W^{ \pm} \ell^{ \pm} \rightarrow 2 \tau+2 j+2 \ell
$$




\begin{tabular}{|c|c|c|c|c|c|c|c|}
\hline \multirow{2}{*}{\multicolumn{2}{|c|}{ Final states }} & \multicolumn{4}{|c|}{ Benchmark Points } & \multicolumn{2}{|c|}{ Backgrounds } \\
\hline & & \multirow{2}{*}{$\frac{\mathrm{BP} 1}{104.6}$} & \multirow{2}{*}{$\begin{array}{l}\mathrm{BP} 2 \\
21.4\end{array}$} & \multirow{2}{*}{$\begin{array}{c}\text { BP3 } \\
78.5\end{array}$} & \multirow{2}{*}{$\begin{array}{l}\mathrm{BP} 4 \\
9444\end{array}$} & \multirow{2}{*}{$t \bar{t} V$} & $V V / V V V$ \\
\hline \multirow{4}{*}{$\begin{array}{l}4 \tau_{\text {jet }}+\not p_{T}^{\prime} \\
\geq 30 \mathrm{GeV}\end{array}$} & $H A$ & & & & & & \multirow{4}{*}{17.1} \\
\hline & $H^{ \pm} H^{\mp}$ & 1.0 & 1.8 & 2.2 & 6.3 & \multirow{3}{*}{0.2} & \\
\hline & $H H^{ \pm}$ & 53.0 & 5.2 & 71.4 & 22.9 & & \\
\hline & $A H^{ \pm}$ & 142.8 & 14.6 & 45.1 & 80.7 & & \\
\hline \multirow{2}{*}{\multicolumn{2}{|c|}{ Total }} & 301.4 & 43.1 & 197.2 & 204.3 & \multirow{2}{*}{\multicolumn{2}{|c|}{$17.3 \pm 2.6$}} \\
\hline & & \pm 25.6 & \pm 10.0 & \pm 11.6 & \pm 24.2 & & \\
\hline \multicolumn{2}{|c|}{$N_{s i g}($ in $\sigma)$} & $\{15.4,18.2\}$ & $\{4.9,6.2\}$ & $\{12.2,14.6\}$ & $\{12.5,14.9\}$ & & \\
\hline \multirow{4}{*}{$4 \tau_{\text {jet }}+1 e$} & $H A$ & 6.7 & 0.0 & 1.2 & 6.8 & \multirow{4}{*}{0.0} & \multirow{4}{*}{0.0} \\
\hline & $H^{ \pm} H^{\mp}$ & 0.4 & 0.1 & 0.3 & 1.1 & & \\
\hline & $H H^{ \pm}$ & 9.7 & 0.5 & 20.7 & 3.3 & & \\
\hline & $A H^{ \pm}$ & 22.7 & 2.6 & 7.3 & 14.0 & & \\
\hline \multicolumn{2}{|c|}{ Total } & $\begin{array}{r}39.5 \\
\pm 5.9\end{array}$ & $\begin{array}{c}3.2 \\
\pm 0.5\end{array}$ & $\begin{array}{l}29.5 \\
\pm 4.4\end{array}$ & $\begin{array}{l}25.3 \\
\pm 3.8\end{array}$ & \multicolumn{2}{|r|}{0.0} \\
\hline \multicolumn{2}{|c|}{$\operatorname{limit}($ in $\sigma$ ) } & 1.9 & 1.2 & 1.8 & 1.7 & & \\
\hline \multirow{4}{*}{$4 \tau_{\text {jet }}+1 \mu$} & $H A$ & 7.5 & 0.0 & 0.8 & 5.6 & \multirow{4}{*}{0.0} & \multirow{4}{*}{0.0} \\
\hline & $H^{ \pm} H^{\mp}$ & 0.1 & 0.4 & 0.4 & 2.0 & & \\
\hline & $H H^{ \pm}$ & 10.3 & 0.6 & 19.0 & 5.4 & & \\
\hline & $A H^{ \pm}$ & 22.5 & 2.1 & 6.1 & 13.2 & & \\
\hline \multirow{2}{*}{\multicolumn{2}{|c|}{ Total }} & 40.4 & 3.1 & 26.3 & 26.1 & \multirow{2}{*}{\multicolumn{2}{|c|}{0.0}} \\
\hline & & \pm 6.1 & \pm 0.5 & \pm 3.9 & \pm 3.9 & & \\
\hline \multicolumn{2}{|c|}{$\operatorname{limit}($ in $\sigma)$} & 1.9 & 1.2 & 1.8 & 1.8 & & \\
\hline \multirow{4}{*}{$4 \tau_{\text {jet }}+2 e$} & $H A$ & 1.3 & 0.0 & 0.2 & 1.6 & \multirow{4}{*}{0.0} & \\
\hline & $H^{ \pm} H^{\mp}$ & 0.0 & 0.0 & 0.0 & 0.1 & & \\
\hline & $H H^{ \pm}$ & 0.5 & 0.0 & 0.1 & 0.3 & & 0.0 \\
\hline & $A H^{ \pm}$ & 0.0 & 0.0 & 0.0 & 0.0 & & \\
\hline Tot & & 1.8 & 0.0 & 0.3 & 2.0 & & 0.0 \\
\hline & $H A$ & 2.0 & 0.0 & 0.2 & 1.4 & & \\
\hline $1 \tau \cdot+2$ & $H^{ \pm} H^{\mp}$ & 0.0 & 0.1 & 0.0 & 0.1 & 0 & 00 \\
\hline $4 \tau_{\text {jet }}+2 \mu$ & $H H^{ \pm}$ & 1.2 & 0.0 & 0.3 & 0.3 & 0.0 & 0.0 \\
\hline & $A H^{ \pm}$ & 0.0 & 0.0 & 0.0 & 0.0 & & \\
\hline Tot & & 3.2 & 0.1 & 0.5 & 1.8 & & 0.0 \\
\hline
\end{tabular}

Table 9. The number of events for $4 \tau_{\text {jet }}+\not p_{T} \geq 30 \mathrm{GeV}, 4 \tau_{\text {jet }}+1 \ell$ and $4 \tau_{\text {jet }}+2 \ell$ final states at $100 \mathrm{fb}^{-1}$ of integrated luminosity at the LHC with $14 \mathrm{TeV}$ center of mass energy. The range for $N_{\text {sig }}$ is calculated incorporating the systematic uncertainties in signal and background events as well. The flavour tagging $(e, \mu)$ has been implemented. 

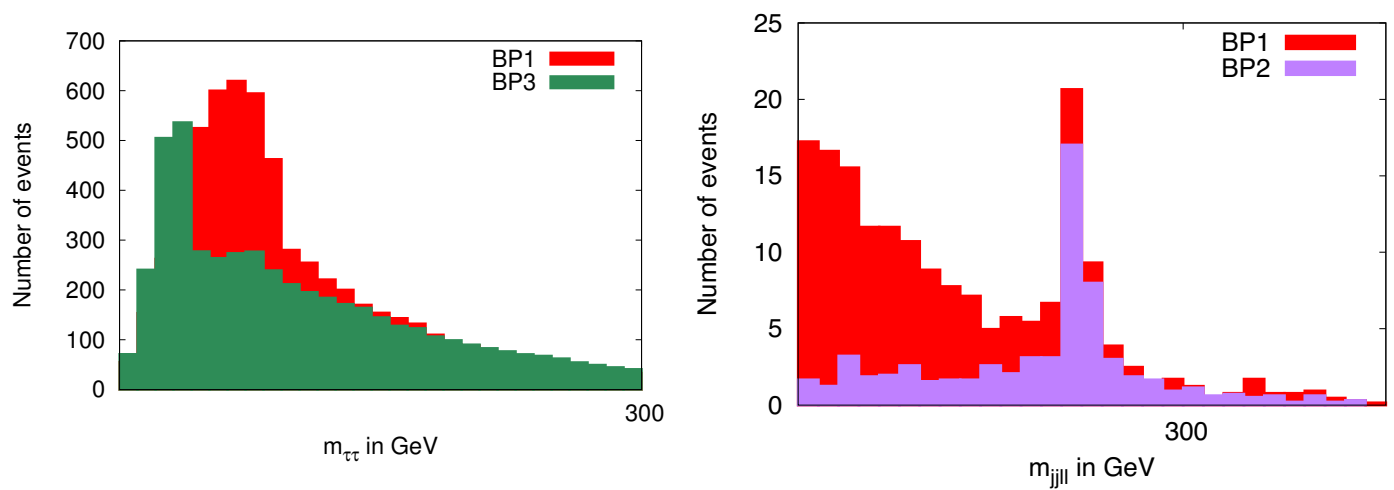

Figure 4. $\tau \tau$ invariant mass distribution (left panel) and $j j \ell \ell$ invariant mass distribution (right panel) for the benchmark points at an integrated luminosity of $100 \mathrm{fb}^{-1}$ at the LHC with $14 \mathrm{TeV}$ center of mass energy.

We reconstruct the light pseudoscalar with $m_{\tau_{\text {jet }}, \tau_{\text {jet }}}$ invariant mass from hadronically reconstructed $\tau$ jets. Figure 4 (left panel) shows the invariant pseudoscalar mass for BP1. Demanding $\left|m_{j j}-m_{W}\right| \leq 10 \mathrm{GeV}$ i.e., the di-jet coming from $W^{ \pm}$boson, we can construct the $W^{ \pm}$and the pseudoscalar $A$ separately. As a next step, we select the events with di-jets from that window and the lepton to construct invariant mass $m_{j j \ell}$. Then we look for the peak of the RHN $N_{i}$ in the invariant mass distribution of $m_{j j \ell}$. Once we get the RHN mass peak, we then construct $m_{j j \ell^{+} \ell^{-}}$, selecting events within $15 \mathrm{GeV}$ of the peak of RHN with the remaining lepton, supposedly coming from the charged Higgs decay. The distribution for BP1 and BP2 are given in figure 4 (right panel) at $100 \mathrm{fb}^{-1}$ of integrated luminosity at the LHC with $14 \mathrm{TeV}$ center of mass energy. It is clearly seen that both of the invariant mass are quite visible at $250 \mathrm{GeV}$. The $\pm 10 \mathrm{GeV}$ window near the peak consists of 30 and 25 events for $\mathrm{BP} 1$ and $\mathrm{BP} 2$, respectively. Interestingly the invariant mass distribution with the demand of $2 \tau+2 \ell$ plus the additional cuts is background free. Thus such points can reconstruct the charged Higgs mass peak with $\lesssim 1000 \mathrm{fb}^{-1}$ integrated luminosity.

For BP3 the major decay modes for the charged Higgs bosons are into $A W^{ \pm}$and $N_{i} \ell_{j}$ but in this case the RHN decays into $A \nu_{i}$. We loose some amount of momentum as missing energy. Furthermore we lose more momentum as missing momentum from tau decays. This spoils the reconstruction of the RHN mass peak and so of the charged Higgs boson via $m_{\tau \tau p \not \ell}$. Nevertheless, the information of the light pseudoscalar from $m_{\tau \tau}$ invariant mass can easily be probed here as well.

\section{Conclusions}

In this article we probe an additional decay channel of the charged Higgs boson decaying into a RHN and a charged lepton. Such non-standard decay mode changes the current lower bound of the charged Higgs boson mass. To be explicit, we have considered Type-X 2HDM, where a light pseudoscalar Higgs boson is still allowed, which opens up additional decay modes of charged Higgs boson to $A W^{ \pm}$and RHN to $A \nu$ states. For relatively heavy pseudoscalar mass we have considered di-tau plus tri-lepton final states with different lepton 
flavour combination. We have shown from a PYTHIA based signal background analysis that $\gtrsim 5 \sigma$ significance can be achieved for all four benchmark points at an integrated luminosity of $100 \mathrm{fb}^{-1}$. For di-tau plus di-lepton signal, such significance can be achieved with very early data at the LHC with $14 \mathrm{TeV}$ center of mass energy. It is interesting to note that the inverse seesaw Yukawa coupling can be probed down to $Y_{N} \sim 0.2$, within $15 \%$ systematic uncertainty, at HL LHC with $3000 \mathrm{fb}^{-1}$ integrated luminosity for this channel. We find that tagging four taus with one lepton (muon or electron) can also reach $5 \sigma$ signal significance for all the benchmark points except BP2. However, the results for $4 \tau+2 \ell$ does not look that promising for any of the benchmark points. Finally we leave it to the experimentalist to calculate the data driven QCD backgrounds, which may contribute via mis-tagging of QCD jets and the subsequent refinement of signal significance, as this is beyond the scope of this analysis.

Next we focus on reconstructing the di-tau invariant mass as shown in figure 4 (left panel). It is evident from the figure that both light and heavy pseudoscalar masses can be reconstructed (BP3 and BP1) here. Followed by that we reconstruct the charged Higgs boson from the decay mode of charged Higgs boson to a RHN plus a charged lepton. We see for $\mathrm{BP} 1$ and $\mathrm{BP} 2$ it is quite possible to reconstruct the charged Higgs boson mass, whereas for BP3 due to large number of missing momentum, viz. neutrinos arising from the decays of RHN and taus, it is not possible to reconstruct such mass peak.

This article thus provides a novel aspect of the charged Higgs boson decaying to RHNs plus a charged lepton. This non-standard decay mode of the charged Higgs boson can be introduced in other types of $2 \mathrm{HDM}$ and supersymmetric models. One can thus use these search strategies to test the respective scenarios.

\section{Acknowledgments}

PB acknowledges IMSc, Chennai and KIAS, Seoul for the visits which were crucial in finishing the project and also SERB CORE Grant CRG/2018/004971. The work of RM has been supported in part by Grants No. FPA2014-53631-C2-1-P, FPA2017-84445-P and SEV-2014-0398 (AEI/ERDF, EU) and by PROMETEO/2017/053 (GV, ES).

Open Access. This article is distributed under the terms of the Creative Commons Attribution License (CC-BY 4.0), which permits any use, distribution and reproduction in any medium, provided the original author(s) and source are credited.

\section{References}

[1] J.F. Gunion and H.E. Haber, The CP conserving two Higgs doublet model: The approach to the decoupling limit, Phys. Rev. D 67 (2003) 075019 [hep-ph/0207010] [INSPIRE].

[2] A. Broggio, E.J. Chun, M. Passera, K.M. Patel and S.K. Vempati, Limiting two-Higgs-doublet models, JHEP 11 (2014) 058 [arXiv:1409.3199] [INSPIRE].

[3] E.J. Chun and J. Kim, Leptonic Precision Test of Leptophilic Two-Higgs-Doublet Model, JHEP 07 (2016) 110 [arXiv: 1605.06298] [INSPIRE]. 
[4] J. Cao, P. Wan, L. Wu and J.M. Yang, Lepton-Specific Two-Higgs Doublet Model: Experimental Constraints and Implication on Higgs Phenomenology, Phys. Rev. D 80 (2009) 071701 [arXiv: 0909.5148] [INSPIRE].

[5] L. Wang and X.-F. Han, A light pseudoscalar of 2HDM confronted with muon g-2 and experimental constraints, JHEP 05 (2015) 039 [arXiv: 1412.4874] [INSPIRE].

[6] T. Abe, R. Sato and K. Yagyu, Lepton-specific two Higgs doublet model as a solution of muon g- 2 anomaly, JHEP 07 (2015) 064 [arXiv:1504.07059] [INSPIRE].

[7] E.J. Chun, Z. Kang, M. Takeuchi and Y.-L.S. Tsai, LHC $\tau$-rich tests of lepton-specific 2HDM for $(g-2)_{\mu}$, JHEP 11 (2015) 099 [arXiv: 1507.08067] [INSPIRE].

[8] E.J. Chun, S. Dwivedi, T. Mondal and B. Mukhopadhyaya, Reconstructing a light pseudoscalar in the Type-X Two Higgs Doublet Model, Phys. Lett. B 774 (2017) 20 [arXiv: 1707.07928] [INSPIRE].

[9] E.J. Chun, S. Dwivedi, T. Mondal, B. Mukhopadhyaya and S.K. Rai, Reconstructing heavy Higgs boson masses in a type $X$ two-Higgs-doublet model with a light pseudoscalar particle, Phys. Rev. D 98 (2018) 075008 [arXiv:1807.05379] [InSPIRE].

[10] P. Bandyopadhyay, E.J. Chun and R. Mandal, Scalar Dark Matter in Leptophilic Two-Higgs-Doublet Model, Phys. Lett. B 779 (2018) 201 [arXiv:1709.08581] [INSPIRE].

[11] J. Li, R. Patrick, P. Sharma and A.G. Williams, Boosting the charged Higgs search prospects using jet substructure at the LHC, JHEP 11 (2016) 164 [arXiv: 1609.02645] [INSPIRE].

[12] M. Guchait and A.H. Vijay, Probing Heavy Charged Higgs Boson at the LHC, Phys. Rev. D 98 (2018) 115028 [arXiv: 1806.01317] [INSPIRE].

[13] B. Coleppa, F. Kling and S. Su, Charged Higgs search via $A W^{ \pm} / H W^{ \pm}$channel, JHEP 12 (2014) 148 [arXiv: 1408.4119] [INSPIRE].

[14] U. Ellwanger, C. Hugonie and A.M. Teixeira, The Next-to-Minimal Supersymmetric Standard Model, Phys. Rept. 496 (2010) 1 [arXiv:0910.1785] [inSPIRE].

[15] P. Bandyopadhyay, K. Huitu and S. Niyogi, Non-standard charged Higgs decay at the LHC in Next-to-Minimal Supersymmetric Standard Model, JHEP 07 (2016) 015 [arXiv:1512.09241] [INSPIRE].

[16] P. Bandyopadhyay and A. Costantini, Distinguishing charged Higgs bosons from different representations at the LHC, JHEP 01 (2018) 067 [arXiv:1710.03110] [INSPIRE].

[17] P. Bandyopadhyay, C. Corianò and A. Costantini, Perspectives on a supersymmetric extension of the standard model with a $Y=0$ Higgs triplet and a singlet at the LHC, JHEP 09 (2015) 045 [arXiv: 1506. 03634] [inSPIRE].

[18] P. Bandyopadhyay, C. Corianò and A. Costantini, Probing the hidden Higgs bosons of the $Y=0$ triplet- and singlet-extended Supersymmetric Standard Model at the LHC, JHEP 12 (2015) 127 [arXiv: 1510.06309] [INSPIRE].

[19] P. Bandyopadhyay, C. Corianò and A. Costantini, General analysis of the charged Higgs sector of the $Y=0$ triplet-singlet extension of the MSSM at the LHC, Phys. Rev. D 94 (2016) 055030 [arXiv: 1512.08651] [INSPIRE].

[20] P. Bandyopadhyay, K. Huitu and A. Sabanci Keceli, Multi-Lepton Signatures of the Triplet Like Charged Higgs at the LHC, JHEP 05 (2015) 026 [arXiv:1412.7359] [INSPIRE]. 
[21] P. Bandyopadhyay, S. Di Chiara, K. Huitu and A.S. Keçeli, Naturality vs perturbativity, $B_{s}$ physics and LHC data in triplet extension of MSSM, JHEP 11 (2014) 062 [arXiv: 1407.4836] [INSPIRE].

[22] P. Bandyopadhyay, K. Huitu and A. Sabanci, Status of $Y=0$ Triplet Higgs with supersymmetry in the light of $\sim 125 \mathrm{GeV}$ Higgs discovery, JHEP 10 (2013) 091 [arXiv: 1306.4530] [INSPIRE].

[23] P. Bandyopadhyay, S. Choubey and M. Mitra, Two Higgs Doublet Type III Seesaw with $\mu-\tau$ symmetry at LHC, JHEP 10 (2009) 012 [arXiv:0906.5330] [INSPIRE].

[24] P. Bandyopadhyay, E.J. Chun and J.-C. Park, Right-handed sneutrino dark matter in U(1)' seesaw models and its signatures at the LHC, JHEP 06 (2011) 129 [arXiv:1105.1652] [INSPIRE].

[25] S. Blanchet, P.S.B. Dev and R.N. Mohapatra, Leptogenesis with TeV Scale Inverse Seesaw in SO(10), Phys. Rev. D 82 (2010) 115025 [arXiv: 1010.1471] [InSPIRE].

[26] P.S.B. Dev and R.N. Mohapatra, TeV Scale Inverse Seesaw in $\mathrm{SO}(10)$ and Leptonic Non-Unitarity Effects, Phys. Rev. D 81 (2010) 013001 [arXiv:0910.3924] [InSPIRE].

[27] H. An, P.S.B. Dev, Y. Cai and R.N. Mohapatra, Sneutrino Dark Matter in Gauged Inverse Seesaw Models for Neutrinos, Phys. Rev. Lett. 108 (2012) 081806 [arXiv:1110.1366] [INSPIRE].

[28] S. Banerjee, P.S.B. Dev, S. Mondal, B. Mukhopadhyaya and S. Roy, Invisible Higgs Decay in a Supersymmetric Inverse Seesaw Model with Light Sneutrino Dark Matter, JHEP 10 (2013) 221 [arXiv: 1306.2143] [INSPIRE].

[29] A. Das, P.S.B. Dev and C.S. Kim, Constraining Sterile Neutrinos from Precision Higgs Data, Phys. Rev. D 95 (2017) 115013 [arXiv:1704.00880] [INSPIRE].

[30] A. Das, Y. Gao and T. Kamon, Heavy neutrino search via semileptonic Higgs decay at the LHC, Eur. Phys. J. C 79 (2019) 424 [arXiv:1704.00881] [INSPIRE].

[31] S. Khalil, TeV-scale gauged B-L symmetry with inverse seesaw mechanism, Phys. Rev. D 82 (2010) 077702 [arXiv: 1004.0013] [INSPIRE].

[32] S. Khalil and A. Sil, Right-handed Sneutrino Inflation in SUSY B-L with Inverse Seesaw, Phys. Rev. D 84 (2011) 103511 [arXiv:1108.1973] [INSPIRE].

[33] P. Bandyopadhyay, E.J. Chun, H. Okada and J.-C. Park, Higgs Signatures in Inverse Seesaw Model at the LHC, JHEP 01 (2013) 079 [arXiv: 1209.4803] [INSPIRE].

[34] T. Hermann, M. Misiak and M. Steinhauser, $\bar{B} \rightarrow X_{s} \gamma$ in the Two Higgs Doublet Model up to Next-to-Next-to-Leading Order in QCD, JHEP 11 (2012) 036 [arXiv:1208.2788] [INSPIRE].

[35] ATLAS and CMS collaborations, Measurements of the Higgs boson production and decay rates and constraints on its couplings from a combined ATLAS and CMS analysis of the LHC pp collision data at $\sqrt{s}=7$ and $8 \mathrm{TeV}$, JHEP 08 (2016) 045 [arXiv:1606.02266] [INSPIRE].

[36] MEG collaboration, New limit on the lepton-flavour violating decay $\mu^{+} \rightarrow e^{+} \gamma$, Phys. Rev. Lett. 107 (2011) 171801 [arXiv:1107.5547] [INSPIRE].

[37] J. Erler and M. Schott, Electroweak Precision Tests of the Standard Model after the Discovery of the Higgs Boson, Prog. Part. Nucl. Phys. 106 (2019) 68 [arXiv: 1902.05142] [INSPIRE].

[38] J. de Blas, Electroweak limits on physics beyond the Standard Model, EPJ Web Conf. 60 (2013) 19008 [arXiv:1307.6173] [InSPIRE]. 
[39] F. del Aguila, J. de Blas and M. Pérez-Victoria, Effects of new leptons in Electroweak Precision Data, Phys. Rev. D 78 (2008) 013010 [arXiv:0803.4008] [InSPIRE].

[40] ATLAS collaboration, Combination of searches for heavy resonances decaying into bosonic and leptonic final states using $36 \mathrm{fb}^{-1}$ of proton-proton collision data at $\sqrt{\mathrm{s}}=13 \mathrm{TeV}$ with the ATLAS detector, Phys. Rev. D 98 (2018) 052008 [arXiv: 1808.02380] [InSPIRE].

[41] CMS Collaboration, Search for heavy Higgs bosons decaying to a top quark pair in proton-proton collisions at $\sqrt{s}=13 \mathrm{TeV}$, CMS-PAS-HIG-17-027 (2017).

[42] F. Staub, SARAH 3.2: Dirac Gauginos, UFO output and more, Comput. Phys. Commun. 184 (2013) 1792 [arXiv:1207.0906] [INSPIRE].

[43] A. Pukhov, CalcHEP 2.3: MSSM, structure functions, event generation, batchs and generation of matrix elements for other packages, hep-ph/0412191 [INSPIRE].

[44] J. Pumplin, D.R. Stump, J. Huston, H.L. Lai, P.M. Nadolsky and W.K. Tung, New generation of parton distributions with uncertainties from global QCD analysis, JHEP 07 (2002) 012 [hep-ph/0201195] [INSPIRE].

[45] ATLAS collaboration, Search for charged Higgs bosons produced in association with a top quark and decaying via $H^{ \pm} \rightarrow \tau \nu$ using pp collision data recorded at $\sqrt{s}=13 \mathrm{TeV}$ by the ATLAS detector, Phys. Lett. B 759 (2016) 555 [arXiv:1603. 09203] [INSPIRE].

[46] ATLAS collaboration, Search for charged Higgs bosons in the $H^{ \pm} \rightarrow$ tb decay channel in $p p$ collisions at $\sqrt{s}=13 \mathrm{TeV}$ using the ATLAS detector, ATLAS-CONF-2016-089 (2016).

[47] CMS collaboration, Search for a light charged Higgs boson decaying to $\mathrm{c} \overline{\mathrm{s}}$ in $p p$ collisions at $\sqrt{s}=8 \mathrm{TeV}$, JHEP 12 (2015) 178 [arXiv:1510.04252] [INSPIRE].

[48] CMS collaboration, Search for charged Higgs bosons with the $\mathrm{H}^{ \pm} \rightarrow \tau^{ \pm} \nu_{\tau}$ decay channel in the fully hadronic final state at $\sqrt{s}=13 \mathrm{TeV}$, CMS-PAS-HIG-16-031 (2016).

[49] ATLAS and CMS collaborations, Charged Higgs searches from ATLAS and CMS, PoS (LHCP2016) 085.

[50] T. Sjöstrand, L. Lönnblad and S. Mrenna, PYTHIA 6.2: Physics and manual, hep-ph/0108264 [INSPIRE].

[51] http://pythia6.hepforge.org/examples/.

[52] M. Cacciari, G.P. Salam and G. Soyez, FastJet User Manual, Eur. Phys. J. C 72 (2012) 1896 [arXiv:1111.6097] [INSPIRE].

[53] CMS collaboration, Performance of reconstruction and identification of tau leptons in their decays to hadrons and tau neutrino in LHC Run-2, CMS-PAS-TAU-16-002 (2016).

[54] G. Bagliesi, Tau tagging at ATLAS and CMS, in 17th Symposium on Hadron Collider Physics 2006 (HCP 2006) Durham, North Carolina, May 22-26, 2006, arXiv:0707.0928 [INSPIRE].

[55] CMS collaboration, CMS technical design report, volume II: Physics performance, J. Phys. G 34 (2007) 995 [INSPIRE].

[56] R. Boniecki, Tau Identification and Reconstruction at the CMS, Acta Phys. Polon. B 44 (2013) 1379 [INSPIRE].

[57] P. Bandyopadhyay, Displaced lepton flavour violating signatures of right-handed sneutrinos in $\mathrm{U}(1)^{\prime}$ supersymmetric models, JHEP 09 (2017) 052 [arXiv: 1511.03842] [INSPIRE].

[58] P. Bandyopadhyay and E.J. Chun, Lepton flavour violating signature in supersymmetric $\mathrm{U}(1)^{\prime}$ seesaw models at the LHC, JHEP 05 (2015) 045 [arXiv: 1412.7312] [INSPIRE]. 